\title{
GIUSTINO II E IL RESTYLING DEL PORTUS NOVUS A COSTANTINOPOLI
}

\section{ALESSANDRO TADDEI}

\author{
UDC: 711.453.4-167(262.53)"o4" \\ 7.078 \\ Preliminary communication \\ Manuscript received: 22. 10. 2015. \\ Revised manuscript accepted: 20. 03. 2016. \\ DOI: 10.1484/J.HAM.5.111332
}

A. Taddei

(Sapienza Università di Roma)

P.le Aldo Moro 5 00185 Roma, Italia

Several monuments, either within or outside the walls of Constantinople, are to be quite certainly attributed to emperor Justin II (565-578). Sources inform us about some public buildings he commanded ex-novo, as well as concerning restorations he undertook in a number of already existing complexes. This latter is the case - for instance - of the old Harbour of Julian, located to the southwest of the hippodrome, in a deep inlet of the coastline of the Sea of Marmara. Having been inaugurated with the name of Portus Novus under emperor Julian (361-363), it provided a convenient shelter even for large ships in the very proximity of the Great Palace. Since it proved to be quite exposed to sand filling, it underwent to frequent dredging works. Further - significant-works were carried out at the harbour by order of Justin II. These works consisted in a whole architectural restyling. New honorary columns were raised, bearing the statues of the emperor and - notably - of his wife Sophia, after whom the harbour was renamed "Harbour of Sophia".

Key words: Early Byzantine Constantinople, Topography, Justin II, Harbour of Julian, Harbour of Sophia, Sea of Marmara

\section{[...] portum quem geminae complexant brachia ripae moenibus adpositis, rapidos contemnere ventos et faciunt, praebentque salum statione quietum: aequoreos frangunt obiecto marmore fluctus et prohibent refluas angustis faucibus undas.}

Così Corippo, nel I libro del suo In laudem Iustini Augusti minoris, descriveva, in maniera quanto più dettagliata la natura retorica del testo gli consentisse, un porto sul quale volgevano lo sguardo il futuro Giustino II (565-578) e la sua consorte Sofia pochi istanti prima che giungesse loro la notizia della morte di Giustiniano, il 14 novembre del $565^{1}$. Come è ormai generalmente accettato, il porto era quello detto "Nuovo", rinnovato e ridecorato da Giustino II.

Quel che si sa dell'antico Portus Novus non è molto ${ }^{2}$. Venne ricavato approfondendo una o due insenature naturali della costa sudorientale della penisola, bagnata appunto dalle acque della Propontide/Mar di Marmara (Fig. 1). Ciò accadde nel corso della seconda metà del IV secolo - in concomitanza con la fondazione di un ulteriore scalo, il porto detto Teodosiano, ubicato ancora più a ovest sul medesimo litorale meridionale della città. La creazione di due bacini di dimensioni relativamente ragguardevoli si rese necessaria infatti al momento in cui gli antichi scali di Byzantion sul Corno d'Oro (soprattutto il Neorion e il Prosphorion) si erano resi ormai insufficienti a fronte dell'incremento nel volume complessivo di traffico commerciale3.

Collocato dalla Notitia urbis nella III regione urbana ${ }^{4}$, leggermente più a ovest del porto palatino del Boukoleon ma non lontano dalla sphendone dell'ippodromo, il Portus Novus si caratterizzava per l'ampia capacità del bacino. Il tardo testo dei Patria $(\mathrm{III}, 37)$, come spesso accade, pretendeva che esso fosse stato costruito già all'epoca di Costantino. Ciò non esclude che Costantino avesse vagheggiato o effettivamente

\footnotetext{
${ }^{1}$ Flavius Cresconius Corippus, In laudem Iustini Augusti minoris, I,104-109, A. CAMERON (ed.), London, 1976, p. 39.

2 Il Portus Novus però - a differenza di altri scali della città - rimase in funzione, nonostante la continua tendenza all'interramento, fino ad epoca tardobizantina, quando la parte occidentale del bacino - ribattezzata con il nome di Kontoskalion - costituiva l'ancoraggio più importante della costa sud. La parte orientale lasciava invece traccia nella toponomastica ottomana dell'epoca classica, quando, a interramento ormai avvenuto, il quartiere che si sviluppò sul suo sito mantenne il nome di Kadırga Limanı (Porto delle Galee). Testi fondamentali per la storia del Porto Nuovo: R. GUILLAND, Les ports de Byzance sur la Propontide, in Byzantion, 23, 1954, pp. 181-238, in part. 195-197. A. CAMERON, Notes on the Sophiae, the Sophianae and the Harbour of Sophia, in Byzantion, 37, 1967, pp. 11-20. W. MÜLLER-WIENER, Bildlexikon zur Topographie Istanbuls. Byzantion-Konstantinupolis-Istanbul bis zum Beginn des 17. Jahrhunderts, Tübingen, 1977, pp. 62-63. ID., Die Häfen von Byzantion Konstantinupolis Istanbul, Tübingen, 1994, pp. 8-9. Più di recente: P. MAGDALINO, The Maritime Neighborhoods of Constantinople, in Dumbarton Oaks Papers, 54, 200o, pp. 209-226. ID., The Harbors of Byzantine Constantinople, in Z. KIZILTAN, G. BARAN ÇELIK (eds.), Stories From The Hidden Harbor: Shipwrecks of Yenikapı, Istanbul, 2013, pp. 11-15. Più in generale, sulla "anatomia" delle coste costantinopolitane, v. C. MANGO, The shoreline of Constantinople in the fourth century, in N. NECIPOĞLU (ed.), Byzantine Constantinople. Monuments, Topography and Everyday Life, Leiden, Boston, Köln, 2001, pp. 17-28,

3 C. MANGO, Le développement urbain de Constantinople (IVe-VIIe siècles), Paris, 1985, p. 39. P. MAGDALINO, Constantinople médiévale : études sur l'évolution des structures urbaines, Paris, 1996, pp. 20-21.

${ }^{4}$ J. MATTHEWS, The Notitia Urbis Constantinopolitanae, in L. GRIG, G. KELLY (eds.), Two Romes: Rome and Constantinople in Late Antiquity, Oxford, 2012, pp. 81-115, in part. p. 88.
} 


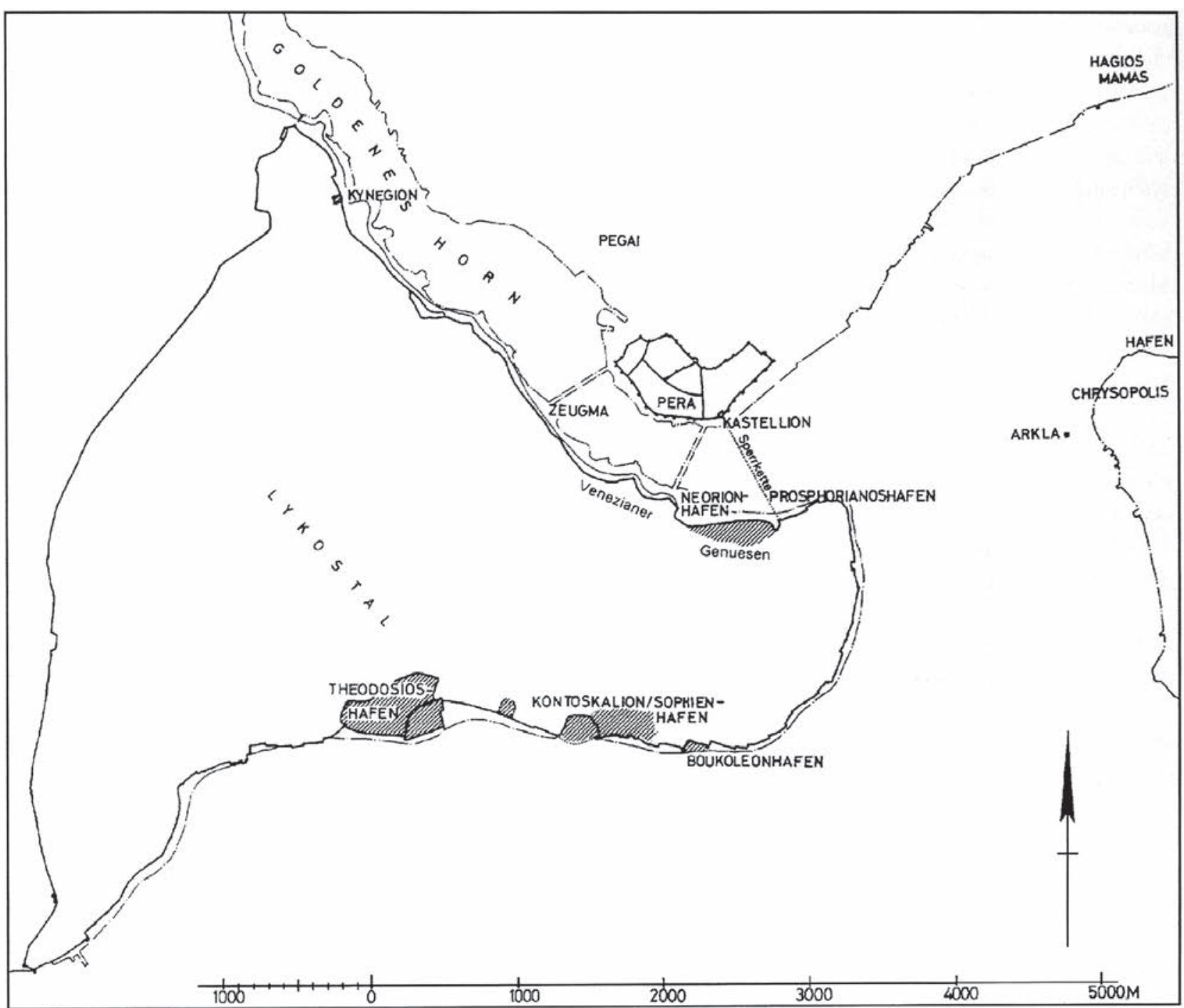

Fig. 1: Pianta di Costantinopoli con indicazione delle aree portuali in epoca tardoantica e bizantina (da W. MÜLLER-WIENER, Die Häfen von Byzantion Konstantinupolis Istanbul, Tübingen, 1994).

disposto la creazione di uno scalo, realizzato però più avanti nel tempo dai suoi successori5.

Il nome di «Portus Iuliani», infatti, che verrà presto a sostituire quello di Portus Novus, ne tramandava la più diffusa attribuzione a Giuliano imperatore (361-363), asseverata da Zosimo ancora all'inizio del VI secolo. Lo storiografo nostalgico della religione tradizionale è debitamente orgoglioso di esaltare la memoria dell'Apostata; ma quest'ultimo, con ogni probabilità, non ebbe neppure il tempo di vedere l'opera finita ${ }^{6}$. Zosimo, che non nutre alcun dubbio sulla paternità dell'impresa, ci descrive il porto come di imponenti dimensioni $^{7}$, realizzato allo scopo di proteggere le navi all'ancora dal forte vento del sud - un'esigenza che si era evidentemente resa molto pressante. Chi giungeva dalla città, prosegue Zosimo, accedeva al porto tramite un «portico di forma più semicircolare che rettilinea» che ne costituiva dunque il monumentale fronte interno ${ }^{8}$. Di Zosimo è debitore, evidentemente, Patria III,37 che insiste sul fatto che il bacino possedesse in origine un prospetto interno a forma di portico

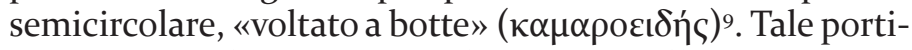
co - qualsiasi fosse la sua conformazione effettiva - doveva quindi probabilmente accompagnare almeno una parte del profilo concavo del bacino portuale (forse quella orientale, cioè verso l'ippodromo) e costituire al tempo stesso sia una

5 Patria Constantinoupoleos, III,37, A. BERGER (ed.), Cambridge (MA), London, 2013, pp. 158-163. Cfr. ID., Untersuchungen zu den Patria Konstantinupoleos, Bonn, 1988, pp. 570-572. Il passo è un riflesso interessante di una tradizione parallela, la quale sostiene l'esistenza, nei tempi più antichi, di un piccolo ancoraggio strettamente legato al complesso residenziale detto di Ormisda (Ta Hormisdou), la cui fama - assai più avanti nel tempo - si deve all'aver ospitato numerosi personaggi legati alla corte imperiale nonché, fra l'altro, il giovane Giustiniano anteriormente alla sua incoronazione nel 527: R. GUILLAND, op. cit. (n. 2), p. 185. W. MÜLLER-WIENER, op. cit. (n. 2), p. 8. L'ancoraggio era forse collocabile presso quello che in epoca ottomana divenne il quartiere della Çatladı Kapı.

${ }^{6}$ J. MATTHEWS, op. cit. (n. 4), p. 101, n. 35. Un curioso passo dei Patria (III,39a) attribuisce il porto non all'imperatore Apostata ma ad un problematico Giuliano consolare (hypatikos), non altrimenti noto: A. BERGER, op. cit. (n. 5), p. 573.

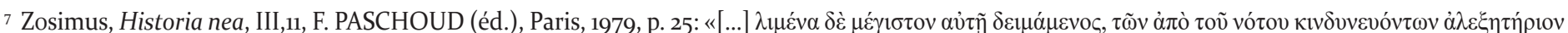

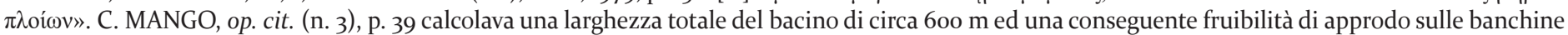
di circa $1000 \mathrm{~m}$.

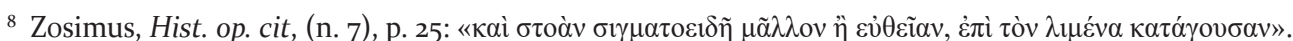

9 Patria Constantinoupoleos, op. cit. (n. 5), p. 160. 
quinta monumentale per chi osservasse dal mare aperto, sia un elemento di separazione e protezione fra l'abitato della III regione e le strutture di servizio dello scalo. E, non a caso, la Notitia urbis introduceva, subito dopo il Portus Novus, il «colonnato semicircolare, che per la sua conformazione viene chiamato con il nome greco di Sigma $»^{10}$. A suggello monumentale del porto si trovava forse una statua di Giuliano, eretta su una base sopraelevata o su una colonna. Fatto sta che Giovanni Malalas ricorda l'abbattimento e la sostituzione di tale statua con una croce, avvenuta al tempo di Giustiniano, nell'anno $553^{11}$.

La natura prettamente semi-residenziale "di prestigio" della zona retrostante il porto, dovuta chiaramente alla vicinanza con le propaggini sudoccidentali del Grande Palazzo trovava già un'eco nella legislazione di Teodosio II. L'imperatore scriveva infatti nel 419 al praefectus urbi Ezio per ordinargli la rimozione dei forni da calce da tutta la zona di costa compresa «inter amphitheatrum et divi Iuliani portum per litus maris», al fine di ristabilire la salubrità dell'aria della città ma, soprattutto, quella dell'adiacente quartiere imperiale ${ }^{12}$. Certo è che la vocazione residenziale delle aree ai margini dei porti di Teodosio e di Giuliano sulla Propontide - a parte i casi precoci, quale le domus di Placidia e Pulcheria ad est dello scalo di Giuliano (I e III regione) e quella di Arcadia presso il teodosiano (IX regione), registrate dalla Notitia ${ }^{13}$ - deve aver rappresentato un fenomeno di lunga durata, se non altro a giudicare dalla presenza di numerosi oikoi aristocratici ben attestati ancora in età mediobizantina ${ }^{14}$.

Nel 464/465 il porto di Giuliano venne colpito - insieme al porto del Neorion sul Corno d'Oro e al foro di Costantino - dal grave incendio che tagliò letteralmente in due la città, devastando otto intere regioni - a sentire il Chronicon paschale - dal Corno d'Oro al Mar di Marmara. Non si ha idea, tuttavia, di quali furono gli effettivi danni subiti, nello specifico, dal porto di Giuliano ${ }^{15}$. L'interramento del bacino, dovuto evidentemente a insufficiente manutenzione, ebbe luogo relativamente presto, tanto che, già nel 509, Anastasio I (491-518) dava ordine di procedere al dragaggio con appositi macchinari da scavo: «Portus Iuliani undis suis rotalibus machinis prius exhaustus caenoque effosso purgatus est $»^{16}$. Il progetto anastasiano prevedeva inoltre la costruzione di nuovi moli ( $\pi \rho \circ \beta$ ó $\lambda o v \varsigma)$, probabilmente a protezione del bacino contro le mareggiate e il costante insabbiamento ${ }^{17}$.

Non è certo se fosse al tempo di Giustiniano, come vorrebbero le Parastaseis, 72 (e, sulla loro scia, i Patria, II,68) o invece più tardi, sotto Giustino II, che il mercato delle derrate marittime fu trasferito dal porto del Neorion proprio al porto di Giuliano ${ }^{18}$. In ogni caso, un secondo incendio ricordato dalla cronaca di Teofane, questa volta nel 56o/561, distrusse diverse abitazioni giungendo dal Porto di Giuliano fino al quartiere detto di Probo ${ }^{19}$. Il disastro aprì la strada a un intervento di restauro dello scalo che dovette essere consistente, tanto da aver lasciato cospicue tracce nelle fonti, e la cui sicura paternità lascia nonostante tutto aperti numerosi interrogativi, solo parte dei quali è stata chiarita nel recente passato dalla critica. Fu Giustino II il responsabile di questa sostanziale operazione.

I versi in epigrafe, estratti dal panegirico In laudem Iustini di Corippo (I,97-114) fanno parte della descrizione del panorama marittimo che Giustino e Sofia ammirano dalla propria dimora $^{20}$. Il testo non esplicita quale sia questa domus o palazzo ove l'aristocratica coppia riceve la notizia della morte dell'imperatore Giustiniano. I due si trovano nella parte superiore di un edificio, all'interno di una sala aperta a loggiato o ad altana, probabilmente rivestita di mosaici «vitrei splendens fulgore metalli $»^{21} \mathrm{e}$ inondata dai raggi solari: «[...] solis dicenda domus». La sala guarda da un lato sul mare «immenso», dall'altro su di uno scalo portuale. A quanto ci dice il panegirista, la coppia ama trattenersi sovente ad osservare lo specchio d'acqua marina e il traffico delle navi «cariche dei commerci dei due mondi»: non stupisce affatto che l'autore insista sulla particolare sensibilità di Giustino per le dimore litoranee e i panorami marittimi. Due tenute marittime suburbane cui Giustino dedicherà la propria attenzione e cura risultano di sua proprietà fin dal tempo in cui egli era stato insignito della cura Palatii. Teofane le conosce sicuramente grazie a una perduta fonte del VI secolo ${ }^{22}$. La prima è un proasteion, nelle immediate vicinanze (letteralmente «nel») porto dell'isola di Prinkipos (Büyükada) ${ }^{23}$. La

1o J. MATTHEWS, op. cit. (n. 4), p. 88. Cfr. M. MUNDELL-MANGO, The Porticoed Street of Constantinople, in NECIPOĞLU (ed.), op. cit. (n. 2), pp. 31-51, in part. p. 37. Cfr. A. BERGER, Regionen und Straßen im frühen Konstantinopel, in Istanbuler Mitteilungen, 47, 1997, pp. 349-414, in part. 361.

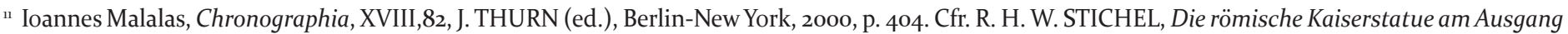
der Antike. Untersuchungen zum plastischen Kaiserporträt seit Valentinian I. (364- 375 v. Chr.), Roma, 1982, p. 31, n. cat. 191. A. BERGER, op. cit. (n. 5), p. 573. F. A. BAUER, Stadt, Platz und Denkmal in der Spätantike, Mainz, 1996, p. 354, n. 80.

${ }_{12}$ Cod. Theod., XIV,6,5, C. PHARR (ed.), New York, 1952, p. 413.

${ }_{13}$ J. MATTHEWS, op. cit. (n. 4), pp. 86, 88, 92. P. MAGDALINO, Maritime Neighborhoods... op. cit. (n. 2), p. 216.

14 P. MAGDALINO, Maritime Neighborhoods... op. cit. (n. 2), p. 214.

${ }^{15}$ Chronicon paschale, M. and M. WHITBY (eds.), Liverpool, 1989, p. 91, n. 296. Per le fonti relativeall'incendiov. A. M. SCHNEIDER, Brände in Konstantinopel, in Byzantinische Zeitschrift, 41, 1941, pp. 382-403, in part. pp. 383-384.

${ }^{16}$ B. CROKE, Count Marcellinus and his Chronicle, Oxford-New York, 2001, p. 24.

${ }_{17}$ Suidae Lexicon, A. ADLER (ed.), Stuttgart, 1989, vol. I, p. 187.

${ }_{18}$ A. CAMERON, J. HERRIN (eds.), Constantinople in the Early Eighth Century: the Parastaseis syntomoi chronikai, Leiden, 1984, pp. 38, 153, 267. Patria Constantinoupoleos, op. cit. (n. 5), p. 98. Sulla data del trasferimento: R. GUILLAND, op. cit. (n. 2), p. 183. A. BERGER, op. cit. (n. 5), p. 430.

19 Theophanes, Chronographia, C. DE BOOR (ed.), Lipsiae, 1883, vol. I, p. 235. Cfr. C. MANGO, R. SCOTT, G. GREATREX (eds.), The Chronicle of Theophanes Confessor. Byzantine and Near Eastern History AD 284-813, Oxford, 1997, pp. 345-346.

${ }^{20}$ Corippus, In laudem Iustini, op. cit. (n. 1), pp. 39, 89, 132-133.

${ }^{21}$ Non penso si possa pensare a pareti o copertura in vetro. Così invece ipotizza nel suo commento Averil Cameron: v. Corippus, In laudem Iustini, op. cit. (n. 1), p. 133 .

${ }_{22}$ Theophanes, Chronographia, op. cit. (n. 19), vol. I, p. 243.

${ }^{23}$ Leo Grammaticus, Chronographia, I. BEKKER (ed.), Bonn, 1842, p. 132. Giorgio Cedreno, probabilmente copiando distrattamente Teofane, cita un altrimenti ignoto «monastero» fondato a Prinkipo da Giustino II: Georgius Cedrenus, I. BEKKER (ed.), Bonn, 1838, vol. I p. 685. 


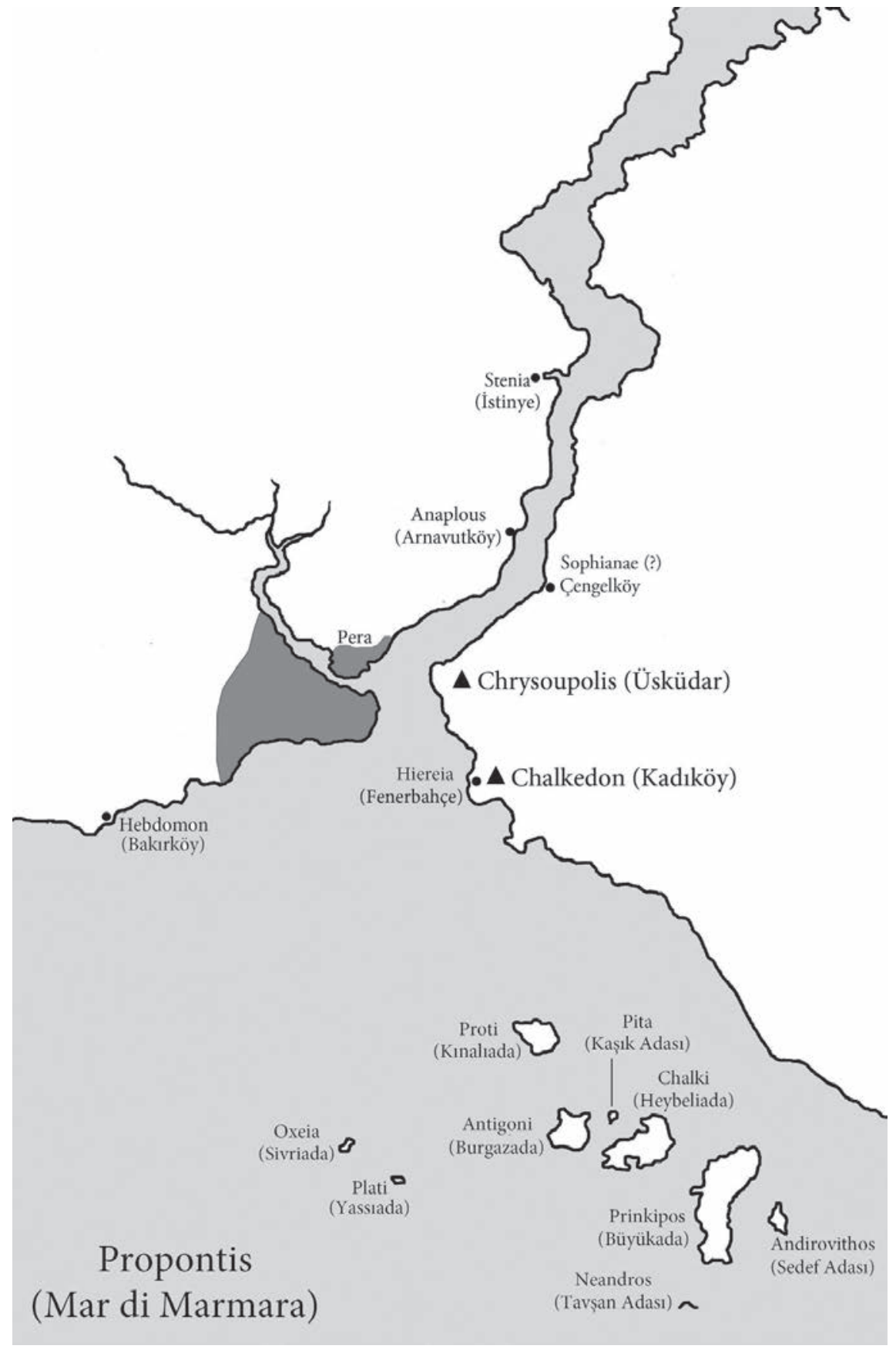

Fig. 2: Il Bosforo e le Isole dei Principi (disegno A. Taddei). seconda, anch'essa un proasteion ma ben più importante, si trova sulla riva asiatica del Bosforo, a nord di Chrysoupolis/Üsküdar, forse sul sito dell'odierna Çengelköy ${ }^{24}$. «Nella chiesa dell'Arcangelo, che ivi si trova» egli aveva peraltro sepolto il figlio Iustus, prematuramente scomparso ${ }^{25}$. In un epigramma databile al 566 lo pseudo-Mariano Scolastico descrive la particolare posizione geografica del proasteion: «dove la terra è tagliata in due dal ventoso canale» vantandone la bellezza del prospetto visto dalla opposta costa europea ${ }^{26}$. La vecchia tenuta sarebbe infatti divenuta, tra il 566 e il 567, lo sfarzoso palation suburbano

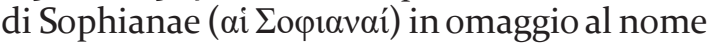
della moglie Sofia (Fig. 2) 27. L'edificio colpisce i contemporanei per il suo sfarzo e gli «splendentia tecta $»^{28}$. Sophianae rimarrà in seguito luogo di villeggiatura privilegiata (prokesson) per i monarchi e vedrà nascere, nel 612, Eraclio Costantino, figlio di Eraclio $(610-641)^{29}$. Ancora all'inizio del IX secolo Teofane ne ricordava la decorazione con marmi preziosi ${ }^{30}$.

Ma il teatro ove si dispiega la scena narrata da Corippo non è il palation del Bosforo. Peraltro, si evince come il punto d'osservazione di Giustino e Sofia non sia molto distante dal bacino portuale cui essi guardano. E per il proasteion di Sophianae, per quanto splendido, non è nota alcuna installazione portuale pubblica, neppure di piccole dimensioni. Lo scenario descritto è quello di uno scalo vasto, trafficato ma efficiente e l'atmosfera solenne del paesaggio, quasi sospesa fuori dal tempo, viene turbata solo dall'improvvisa notizia del decesso di Giustiniano. Non solo: la lunga scena si dipana in una residenza (domus/oikos) da cui la coppia raggiungerà facilmente il cuore del Grande Palazzo, nella notte: «itur in arcem [...] incedunt media securam nocte per urbem» $(\mathrm{I}, 187-190)$. Immediata conseguenza di tale assunto è che la domus in questione dovesse esistere già alla data della salita al trono di Giustino, nel 565 e si è ritenuto fosse quella detta Sophiae ( $\alpha$ i

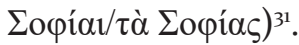

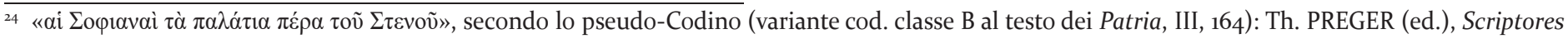
originum constantinopolitanarum, vol. II, Leipzig, 1907 p. 267. E sarà proprio la predilezione particolare per la riva asiatica del Bosforo, a portare infine Giustino II a restaurare il proasteion giustinianeo di Hiereia (oggi Fenerbahçe): Patria Constantinoupoleos, op. cit. (n. 5), pp. 208-209. Su Hiereia v. J. PARGOIRE, Hiéria, in Izveštiâ Russkago arheologičeskago instituta v’ Konstantinopolě, 4/2, 1899, pp. 7-78.

25 Theophanes, Chronographia, op. cit. (n. 19), vol. I, p. 243. Identificazione Sophianae-Cengelköy in J. PARGOIRE, op. cit. (n. 24), p. 43. R. GUILLAND, op. cit. (n. 2), p. 192 riteneva potesse trattarsi del santuario micaelico del Sosthenion, che però si trova sull'opposta riva europea, collocato da taluni presso Stenia/İstinye, da altri presso Anaplous/Arnavutköy. In entrambi i casi, rimarrebbe senza soluzione il problema rappresentato dal fatto che il passo di Teofane fa riferimento esplicitamente ad una vicinanza tra la chiesa e la tenuta di Giustino. Cfr. C. MANGO, R. SCOTT, G. GREATREX (eds.), op. cit. (n. 19), p. 358 n. 2. Si veda anche ibid., p. 602 n. 13 ove si propone, in alternativa, di riconoscere Sophianae nei ruderi di epoca bizantina presenti un tempo nell'area di Beylerbey.

${ }^{26}$ Anthologia Palatina, IX.657, F. CONCA, M. MARZI (a cura di), vol. II, Torino, 2009, pp. 542-543, con attribuzione ad Agazia Scolastico). Sulla data dell'epigramma, analisi in A. CAMERON, Notes on the Sophiae, pp. 15-17.

${ }_{27}$ Theophanes, Chronographia, op. cit. (n. 19), vol. I, p. 243): datazione al 569. Contra, si veda la precisazione cronologica di A. CAMERON, Notes on the Sophiae... op. cit. (n. 2), pp. 11-13 basata sulla cronologia di pubblicazione del IV libro del panegirico di Corippo: Corippus, In laudem Iustini, op. cit. (n. 1), pp. 81, 205.

${ }_{28}$ Corippus, In laudem Iustini, IV, 287 op. cit. (n. 1), pp. 81.

${ }_{29}$ Chronicon paschale, op. cit. (n. 15), p. 154, n. 431.

30 Theophanes, Chronographia, op. cit. (n. 19), vol. I, p. 243).

${ }^{31}$ A. CAMERON, Notes on the Sophiae... op. cit. (n. 2), pp. 14-15.
} 
Di tale residenza, appartenente al gruppo degli oikoi aristocratici che gravitavano sul litorale della III regione, non troppo lontano dall'ippodromo e dal quartiere di Hormisdas, è sì abbondante traccia nelle fonti, ma con le datazioni e attribuzioni le più varie. Teofane la dice addirittura costruita da Tiberio II (578-582) come residenza semi-coatta per Sofia ormai vedova, nel $579^{32}$; ma sappiamo che l'ex-imperatrice rifiutò il più a lungo possibile di lasciare i propri appartamenti del grande Palazzo e fu, al contrario, Tiberio a dover riorganizzare Hormisdas per alloggiare la propria famiglia ${ }^{33}$. I Patria, III,125 parlano di una ricostruzione o restauro

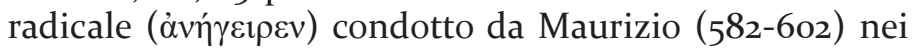
primi anni del suo regno, al fine di riadattare la residenza di Sofia - definita «vecchia» - per la suocera Anastasia, figlia di Tiberio ${ }^{34}$. In questo caso, almeno, la retrodatazione della costruzione originaria agli ultimi anni di Giustiniano assegnerebbe effettivamente all'aggettivo «vecchia» 0 «antica» un senso maggiore.

Nel suo commentario a Corippo, Averil Cameron mise opportunamente in relazione i versi 97 e ss. con l'importante aneddoto contenuto in Patria III,37 subito dopo la descrizione delle origini del Porto di Giuliano ${ }^{35}$. È il punto in cui l'anonimo si dispone a spiegare come il restauro del bacino sia avvenuto per iniziativa di Sofia, che viene qui detta augusta, poiché Patria III,37 intende esplicitamente collocare l'episodio dopo la presa di potere dell'ex-kouropalates Giustino (cfr.

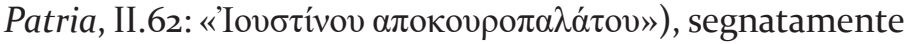
nel $569^{36}$. Sofia è detta guardare con angoscia, anche questa volta da un'altana (heliakon), le faticose manovre delle navi che intendono attraccare al porto, esposte alla forza dei venti e delle onde. Il brano appena citato di Patria III,37 aveva condotto Guilland a concludere che il bacino interno del Porto di Giuliano doveva essersi nuovamente e in gran parte insabbiato e che soltanto i moli esterni fossero ancora disponibili per le navi di pescaggio e portata maggiore ${ }^{37}$. C’è da osservare che Patria III,37 compila confusamente materiali ben più antichi, cronachistici e non, preoccupandosi più che altro di evidenziare l'iniziativa di Sofia come causa prima del nuovo nome del porto. Il testo, con la sua curiosa rielaborazione di Corippo, riflette ciononostante la realtà storica di una pratica di collaborazione coniugale in materia di committenza che diverrà la norma per tutto il regno di Giustino II. Sofia chiede al marito di trovare i fondi neces-

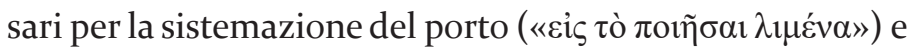
Giustino accondiscende volentieri. Il porto viene appunto

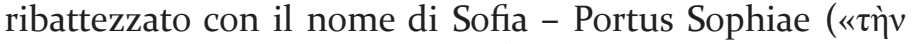

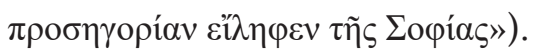

Patria, III,37 fornisce una volta di più ulteriori particolari del restauro di Giustino II, il quale: «[...] diede ordine al patrikios e praepositos Narsete nonché al suo protovestiarios Troilo di costruire il porto. I due lo realizzarono per mezzo dello scavo di una grande fossa» Il che fa pensare che il bacino di Giuliano potesse essere stato ampliato. Meno solenne è invece il cd. Leone Grammatico (sec. X-XI),

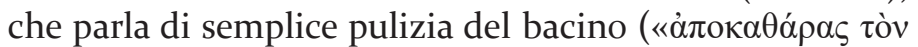
$\lambda \mu \varepsilon \dot{v} v \propto)$, avvenuta, secondo la sua scansione temporale, dopo l'elevazione a cesare di Tiberio II, dunque nel $574^{38}$. Dei due personaggi citati dai Patria, quello storicamente attestato è soltanto il primo: Narsete $(+581)^{39}$, divenuto fin dal 565 membro della guardia personale di Giustino II, otterrà le cariche di spatharios e sakellarios, con le quali "firma" un'epigrafe, verosimilmente anteriore al 574 , commemorante il restauro di un tratto dell'antemurale teodosiano presso l'odierna Mevlevihane Kapi ${ }^{40}$. Il secondo, Troilo, forse un comes sacrae vestis di Giustino II - protovestiarios è palese anacronismo - rimane di dubbia autenticità ${ }^{4}$.

Ma quando avvenne effettivamente la risistemazione del porto? Quella che Corippo intendeva celebrare, indirettamente e senza dichiararlo in termini espliciti, era la rinnovata veste del Porto di Giuliano. Bisogna ammettere che Corippo abbia voluto anacronisticamente - e a scopo prettamente laudatorio - introdurre nella celebrazione della presa di potere da parte del kouropalates Giustino un'opera realizzata nei fatti dopo tale accessione, a differenza dello oikos di Sofia, che può benissimo aver rappresentato un momento di committenza privata ante incoronazione. Se infatti si decidesse di ritenere verosimile un restauro del porto da parte di un semplice curator Palatii, il quale, come Averil Cameron giustamente notava, non venne mai designato ufficialmente come proprio successore dallo zio Giustiniano $\mathrm{O}^{42}$, bisognerebbe fare ricorso alla larga disponibilità di conoscenze, mezzi e libertà di azione di cui Giustino certo godeva negli ultimi anni del regno del predecessore, ma tale appiglio non sembra assolutamente reggere. La cronologia, a ben vedere, non lascia vasti margini: il libro I di Corippo risale con ogni probabilità agli ultimi mesi del 566, cioè neanche un anno dopo l'incoronazione ${ }^{43}$. Bisogna dunque pensare che i lavori di Giustino non consistessero in un'opera di ampliamento dello scalo - che avrebbe comportato costi enormi e lunghi tempi di realizzazione - ma in una radicale azione di manutenzione e restyling, dettata dal desiderio del nuovo sovrano di lasciare in tempi brevi, e per quanto possibile dopo la stagione architettonica giustinianea, la propria impronta a uno dei luoghi chiave della città.

\footnotetext{
32 Theophanes, Chronographia, op. cit. (n. 19), vol. I, p. 250). R. GUILLAND, op. cit. (n. 2), p. 193.

33 A. CAMERON, The Empress Sophia, in Byzantion, 45/1, 1975, pp. 5-21, in part, p. 19.

34 Patria Constantinoupoleos, op. cit. (n. 5), pp. 192-193.

35 A. CAMERON, Notes on the Sophiae... op. cit. (n. 2), p. 14. EAD., Patronage of Justin II... op. cit. (n. 2), p. 72.

${ }^{36}$ Patria Constantinoupoleos, op. cit. (n. 5), p. 163, n. 53: «252 anni dopo la fondazione di Costantinopoli», dove $0=317$.

37 R. GUILLAND, op. cit. (n. 2), pp. 183, 186-187.

${ }^{38}$ Leo Grammaticus, Chronographia, op. cit. (n. 23), p. 135.

39 J. R. MARTINDALE, The Prosopography of the Later Roman Empire, vol. III/2 (AD 527-641), Cambridge, 1992, pp. 930-931, s.v. Narses 4 (d'ora in poi: PLRE). Su Narsete spatharios, cfr. inoltre C. MANGO, R. SCOTT, G. GREATREX (eds.), op. cit. (n. 19), p. 360 n. 1.

$4^{\circ}$ N. ASUTAY-EFFENBERGER, Die Landmauer von Konstantinopel-Istanbul, Berlin-New York, 2007, pp. 173-174 (con bibliografia).

${ }^{41}$ A. CAMERON, Patronage of Justin II... op. cit. (n. 2), p. 7o. PLRE, III/2, p. 1376, s.v. Troilus 2.

${ }^{42}$ A. CAMERON, op. cit. (n. 33), pp. 6-7.

43 Corippus, In laudem Iustini, op. cit. (n. 1), p. 2.
} 


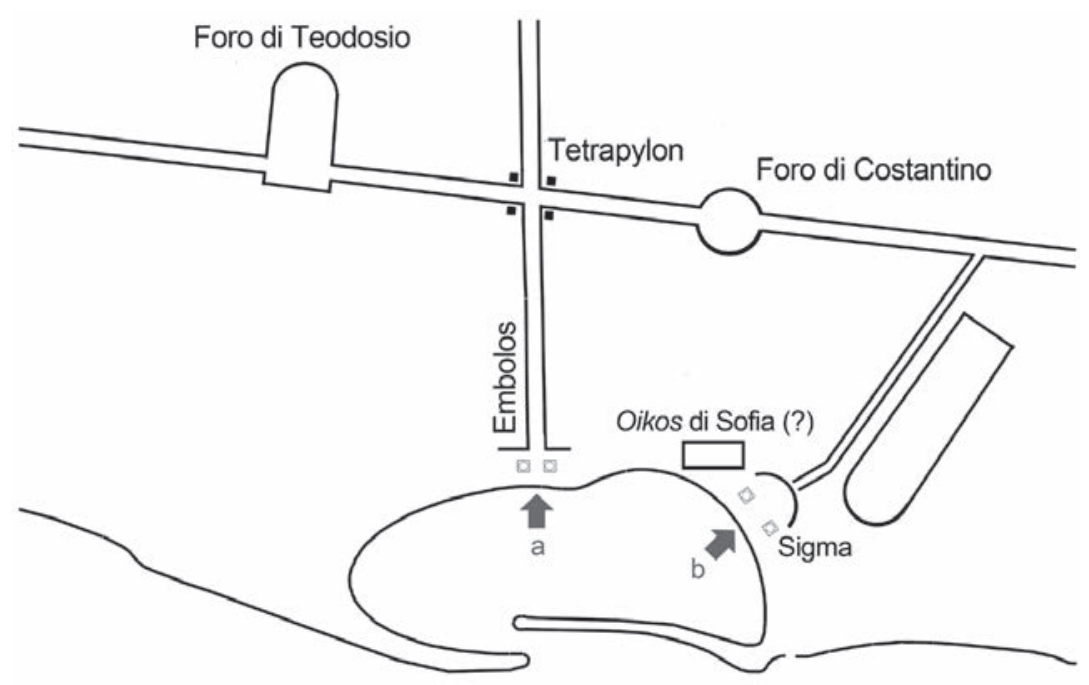

0 tivamente dedicate a Giustino e a Sofia ${ }^{46}$. Sulla base di tali contrastanti affermazioni è difficile giungere a delle conclusioni sulla collocazione dei gruppi statuari del Porto di Sofia. Cameron accetta come maggiormente probabile l'esistenza di almeno due gruppi distinti di statue con effigi di membri della famiglia di Giustino II, la cui combinazione avrebbe consentito di raggiungere il massimo impatto scenografico, così come si era probabilmente fatto per il gruppo di tre - Sofia-Arabia-Elena (quest'ultima nipote di Sofia) - collocato sul Milion ${ }^{47}$.

L'esistenza di gruppi statuari "di famiglia" al Porto di Sofia potrebbe anche essere stata indipendente dal progetto di restauro di Giustino II. L'indizio della presenza di colonne come sostegno delle statue citate dai Patria è significativo, in quanto dice qualcosa di più sulla conformazione di base dello spazio monumentale del porto. Martina Jordan Ruwe aveva giustamente colto la necessità di ipotizzare due soluzioni distinte ${ }^{48}$, a seconda che si ritenesse affidabile il dettato dei Patria, dunque un tetrakionion, o si preferissero i due più tardi storiografi, i quali, nel limitare il numero

Fig. 3: Pianta dell'area del Porto di Giuliano/Sofia con indicazione delle possibili posizioni dei monumenti a colonna (disegno A. Taddei).

Quanto alla articolazione del nuovo Porto di Sofia e alla sua decorazione, pochissimi sono gli elementi a noi noti, a parte quelli estrapolabili dal panegirico di Corippo: la forma dello scalo ricorda senza dubbio la precedente fase anastasiana del porto di Giuliano, con le due banchine simmetriche "a tenaglia", che lasciano fra loro solo una stretta imboccatura; le due banchine appaiono adesso rinforzate da muraglioni in blocchi di marmo, appositamente creati per contrastare efficacemente la forza delle mareggiate, offrire un luogo di ancoraggio sicuro e scongiurare un troppo rapido processo di insabbiamento.

Non abbiamo alcuna informazione sull'architettura degli edifici portuali, di rappresentanza o di servizio. Le fonti invece insistono unicamente nel registrare un ricco "set" di statue su colonne onorarie approntato per celebrare Giustino II, ma non vi è accordo neppure sull'effettiva articolazione di quest'ultimo; per Patria III,37: «Al centro del porto [Giustino] fece erigere quattro statue al di sopra di altrettante colonne, una di Sofia, una di Arabia sua figlia, una dell'imperatore Giustino [sic] e una di Narsete che aveva sovrinteso [ai lavori]» ${ }^{44}$ Patria II,62 (che in modo confuso "anticipava" il passo III,37) vedeva al posto dell'effigie di Narsete quella di Vigilantia, sorella di Giustiniano e madre di Giustino

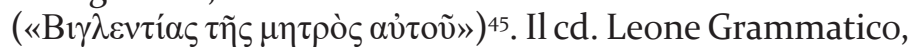
seguito da Giorgio Cedreno, parla di due sole colonne, rispetdelle colonne a due potrebbero tuttavia aver attinto a una fonte più degna di fede di quanto non lo siano i confusi aneddoti dei Patria stessi. La soluzione a quattro colonne, nonostante la sua imponenza, sembra la meno probabile, se non per l'incertezza dei personaggi effigiati (Narsete sostituito da Vigilantia, Arabia), per l'eccessiva importanza che tale articolazione avrebbe conferito a soggetti "minori" in un contesto tanto significativo quanto quello di uno dei maggiori scali marittimi della città. Nessuna memoria di un tetrakionion familiare di Giustino II sembra emergere dalle altre fonti a nostra disposizione. Al contrario, la erezione di monumenti "doppi", di Giustino II e Sofia, ove l'imperatrice è innalzata esattamente allo stesso onore e prestigio del marito - «duo lumina mundi» - rappresenta una costante dell'arte in funzione propagandistica del nipote di Giustiniano e, soprattutto un sintomo della rilevanza e visibilità politica della ambiziosa e capace consorte ${ }^{49}$. Rudolf Stichel aveva potuto catalogare almeno tre casi di monumenti doppi (tutti perduti, esattamente come quelli del porto): due statue erette da Giuliano, praefectus urbi nel 566, di fronte alla facciata delle terme di Zeuxippo ${ }^{50}$; altre due splendenti di bronzo - poste forse su alte basi o colonne - che decoravano un contesto residenziale eretto dall'imperatore nella parte nordoccidentale della città, oggi generalmente collocato nel quartiere del Deuteron ${ }^{51}$; o, ancora, la coppia di agalmata

\footnotetext{
${ }_{44}$ Patria Constantinoupoleos, op. cit. (n. 5), pp. 160-163. Arabia sarebbe Figlia di Giustino II. C. MANGO, Notes d'épigraphie et d'archéologie. Constantinople, Nicée, in Travaux et Mémoires, 12, 1994, pp. 344-345 ne metteva in dubbio, se non la realtà storica, il nome, noto solamente da testi patriografici o agiografici tardivi, sui quali v. PLRE, III/1, p. 102.

45 PLRE, III/2, p. 1376.

${ }^{46}$ Leo Grammaticus, Chronographia, op. cit. (n. 23), p. 135. Georgius Cedrenus, op. cit. (n. 23), vol. I, p. 685.

${ }^{47}$ A. CAMERON, Patronage of Justin II... op. cit. (n. 2), p. 70.

${ }_{48}^{8}$ M. JORDAN-RUWE, Das Säulenmonument: zur Geschichte der erhöhten Aufstellung antiker Porträtstatuen, Bonn, 1995, pp. 186-187.

49 A. CAMERON, op. cit. (n. 33), pp. 8-9. EAD., Patronage of Justin II... op. cit. (n. 2), p. 71. Si veda inoltre il recente contributo di J. RADLEGGER, Die Stifterinnentätigkeit der Kaiserin Sophia: Impuls für die Gleichberechtigung mit dem Kaiser?, in Wiener Jahrbuch für Kunstgeschichte, 6o-61, 2011-2012, pp. 109-119, in part. 114-119.

${ }_{50}$ Anthologia Palatina, op. cit. (n. 26), pp. 608-611 (IX,803-804). R. H. W. STICHEL, op. cit. (n. 11), p. 112 (n. cat. 134). Sul personaggio di Giuliano, menzionato, fra l'altro, da Corippo in occasione del ristabilimento del consolato da parte di Giustino II (566), si veda Alan CAMERON, Some prefects called Julian, in Byzantion, 47, 1977, pp. 42-64, in part. 56-57. Cfr. PLRE, III/1, pp. 735-736, s.v. Iulianus 15.

${ }^{51}$ Iohannis Ephesini Historiae ecclesiasticae. Pars tertia, E. W. BROOKS (ed.), Louvain, 1952, p. 111 (testo siriaco p. 151): «palatium ... in Astydeutero (?)». Cfr. R. H. W. STICHEL, op. cit. (n. 11), p. 112 (n. cat. 135). Ė il «palation to deuteron», che Teofane, sulla scorta di Giovanni di Efeso, dice essere stato risis-
} 


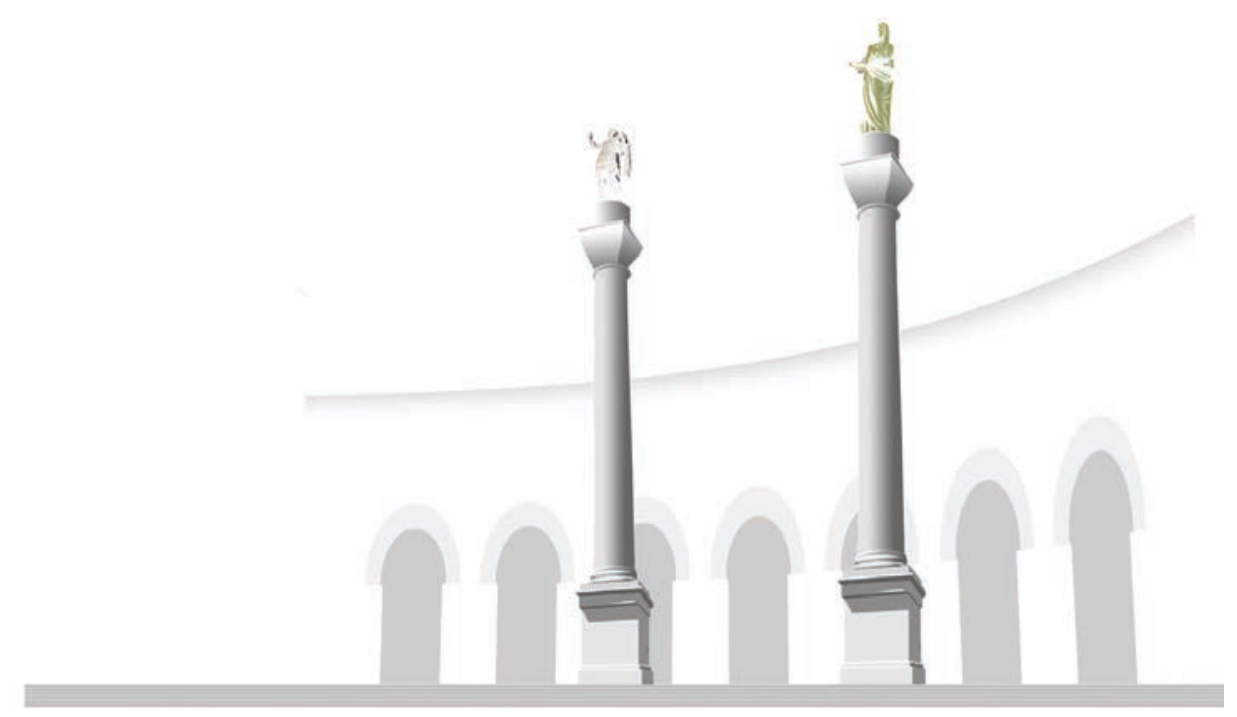

Fig. 4: Ricostruzione ipotetica dei due monumenti a colonna del Porto di Giuliano/Sofia (disegno A. Taddei).

della coppia imperiale innalzati dal praefectus Domnino davanti al Praetorium ${ }^{52}$.

A ragione Martina Jordan-Ruwe ha sottolineato la possibilità che due statue, montate su colonne onorarie al centro del porto, rappresentassero il punto di partenza scenico ideale per un'arteria diretta dal porto verso la Mese (probabilmente, il tratto meridionale, in salita relativamente pronunciata, del cd. Makros Embolos fiancheggiato dal Portico di Domnino $)^{53}$ ma anche un segnacolo per chi osservasse dal mare (Fig. 3: lettera a).

A questo punto però, viene da chiedersi se al momento della erezione delle colonne, il vecchio Sigma, il portico semicircolare dell'impianto giulianeo e anastasiano, esistesse ancora o fosse andato distrutto nella ristrutturazione del porto. Nel caso esso fosse ancora in piedi, poteva offrire ancora, come si è appreso da Zosimo all'inizio del VI secolo,

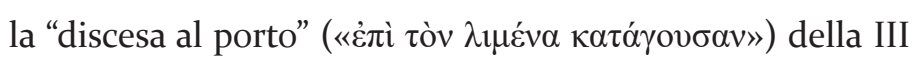
regione ${ }^{54}$, verosimilmente, dunque, dal lato dell'ippodromo. Allora piace immaginare che i monumenti onorari di Giustino e Sofia si inserissero in simmetria con il Sigma (Fig. 3: lettera b), e fossero idealmente abbracciati dalla stoa stessa, la quale poteva, a sua volta, ospitare altri gruppi statuari, comequelli della famiglia imperiale e del sakellarios Narsete, confusamente ricordati dai Patria.

Se ciò fosse dimostrabile, si potrebbe delineare una facciata monumentale con colonne onorarie gemelle e portico sul fondale (Fig. 4), collocata sulla direttrice visiva dell'ippodromo e dei settori circostanti del Grande Palazzo, tra i quali l'oikos di Sofia, svettanti grazie al rapido innalzarsi delle quote del suolo, in un insieme perfettamente godibile da chi giungesse navigando o stazionasse sulla banchina interna del porto.

temato nell'anno 569/570 sul terreno di una vecchia proprietà del kouropalates Giustino: C. MANGO, R. SCOTT, G. GREATREX (eds.), op. cit. (n. 19), p. 359. C. MANGO, The Art of the Byzantine Empire 312-1453, Englewood Cliff (NJ),1972, p. 124, n. 7. A. CAMERON, Notes on the Sophiae... op. cit. (n. 2), pp. 17-18. Cfr. EAD., Patronage of Justin II... op. cit. (n. 2), p. 72. Al Deuteron di Giustino II è stata collegata la colonna marmorea monumentale che Cristoforo Buondelmonti, Pierre Gilles ed altri videro ancora in piedi sulla quinta collina, alla distanza di quattro stadi dai SS. Apostoli, e che venne reimpiegata nella prima metà del XVI secolo nella fabbrica della Süleymaniye Camii. Fatta salva la compatibilità della colonna con il quartiere del Deuteron, il fatto che la colonna potesse in origine recare o meno una statua di Giustino II (una delle due statue bronzee della Historia di Giovanni di Efeso), rimane questione assai complessa da risolvere, condizionata come è da una ormai irrimediabilmente intricata attività esegetica applicata a fonti della più varia origine. L'esistenza di una «colonna di Giustino II al Deuteron» mi sembra data per acquisita nella recente e articolata esposizione di N. ASUTAY-EFFENBERGER, A. EFFENBERGER, Die 'columna virginea' und ihre Wiederverwendung in der Süleymaniye Camii, in Millennium, 1, 2004, pp. 369-407, in part. 377-381, cui si fa necessariamente riferimento anche per la imponente bibliografia precedente.

${ }^{2}$ Anthologia Palatina, IX.812-813, op. cit. (n. 26), pp. 606-607. R. H. W. STICHEL, op. cit. (n. 11), pp. 112-113 (n. cat. 135, 137) con riferimento alle relative fonti. Averil CAMERON, Alan CAMERON, The Cycle of Agathias, in Journal of Hellenic Studies, 86, 1966, pp. 6-25, in part. 21. A. CAMERON, Patronage of Justin II... op. cit. (n. 2), pp. 71-72, n. 42. Domninus fu probabilmente il successore di Giuliano alla carica di praefectus urbi, nel 567: PLRE, III/1, pp. 416-417, s.v. Domninus 2.

53 JORDAN-RUWE, op. cit. (n. 48), p. 187. Per l'Embolos, si veda C. MANGO, op. cit. (n. 3), pp. 30-32. M. MUNDELL-MANGO, The Commercial Map of Constantinople, in Dumbarton Oaks Papers, 54, 2000, pp. 189-208, in part.194, 197. EAD., op. cit. (n. 10), pp. 31 , 39 (con bibliografia).

54 Zosimus, Hist., op. cit. (n. 7), p. 25 (III, 11). 